5. Nienaber CA, Kodolitsch YV, Nicolas V, Siglow V, Piepho A, Brockhoff $\mathrm{C}$, et al. The diagnosis of thoracic aortic dissection by noninvasive imaging procedures. $N$ Engl J Med. 1993;328:1-9.
6. Penco M, Paparoni S, Dagianti A, Fusilli C, Vitarelli A, Remigis FD, et al. Usefulness of transesophageal echocardiography in the assessment of aortic dissection. Am J Cardiol. 2000;86:53G-6G.

\title{
Flap suffocation: An uncommon mechanism of coronary malperfusion in acute type $A$ dissection
}

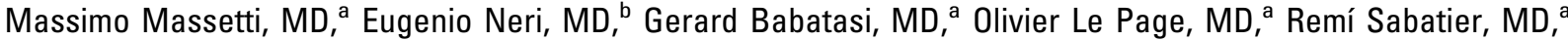 \\ Dimitrios Buklas, MD, ${ }^{a}$ Gilles Grollier, $\mathrm{MD}^{\mathrm{a}}$ and André Khayat, MD, ${ }^{\mathrm{a}}$ Caen, France, and Siena, Italy
}

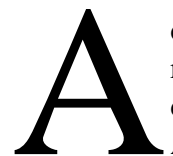

cute myocardial ischemia or infarction as a result of retrograde coronary artery dissection is a well-known complication after acute type A aortic dissection. ${ }^{1}$ Although the prevalence of chronic coronary artery disease among patients with acute dissection of the ascending aorta has been reported to be between $8 \%$ and $41 \%,{ }^{2}$ retrograde dissection of the aortic root reaching the coronary ostia is the most typical cause of acute coronary malperfusion observed in this condition. ${ }^{1}$ However, other mechanisms of myocardial ischemia, related to the diastolic expansion of the false aortic channel, have been described by Borst. $^{3}$

We present here the case of a patient with acute type A aortic dissection in whom an acute coronary malperfusion was sustained by an atypical mechanism of flow obstruction.

\section{Clinical Summary}

A 49-year-old man with a history of hypertension and sarcoidosis was transferred from an outlying hospital to our institution in severe cardiogenic shock. Two hours previously, he had experienced the sudden onset of acute chest pain, followed within a few minutes by shortness of breath and severe hypotension. A 12-lead electrocardiogram showed diffuse ST-segment depression and Twave inversion with an anterior ST elevation (without a related Q wave). A transthoracic echocardiogram was not of diagnostic quality because of a poor acoustic window. Intravenous heparin, inotropic support, and mechanical ventilation had been given without any change in the patient's condition. Systemic fibrinolysis was administered without improvement. At the time of arrival at

From the Thoracic and Cardiovascular Surgery Department, University Hospital of Caen, France, ${ }^{a}$ and the Vascular Surgery Department, University Hospital of Siena, Italy. ${ }^{\text {}}$

Received for publication Aug 10, 2002; accepted for publication Sept 9, 2002.

Address for reprints: Massimo Massetti, MD, Thoracic and Cardiovascular Surgery Department, University Hospital, 14033, Caen, France (E-mail: massetti-m@chu-caen.fr).

J Thorac Cardiovasc Surg 2003;125:1548-50

Copyright $\odot 2003$ by The American Association for Thoracic Surgery $0022-5223 / 2003 \$ 30.00+0$

doi:10.1016/S0022-5223(03)00047-3 our institution, the patient had a blood pressure of $68 / 45 \mathrm{~mm} \mathrm{Hg}$ and an irregular pulse of 110 beats/min. The peripheral pulses were weak but symmetric. Preoperative creatine kinase MB levels were not significantly elevated.

Transesophageal echocardiography was performed to obtain an analysis of the left ventricular segmental wall motion before urgent coronary angiography; surprisingly, it demonstrated the presence of an intimal flap in the ascending aorta with a circumferential distal disruption of the inner aortic wall layers. The cylindric flap presented a proximal invagination movement through the aortic valve, inside the left ventricle, during diastole (Figure 1). This diastolic intussusception of the tubular flap determined the flow obstruction of the coronary ostia and the subsequent myocardial malperfusion. Global biventricular wall motion abnormalities and a left ventricular ejection fraction of 0.25 were demonstrated. We decided to operate on the patient without delay.

The chest was opened through a median sternotomy, and the pericardium was entered. Minimal bloody pericardial effusion was detected. Severe biventricular distention and global hypokinesia were present. The dilated ascending aorta consisted of only a thin, bluish layer of tissue; the dissection terminated $2 \mathrm{~cm}$ proximal to the origin of the brachiocephalic trunk. Mildly hypothermic $\left(32^{\circ} \mathrm{C}\right)$ cardiopulmonary bypass was instituted between the right atrium and right axillary artery; the ascending aorta was crossclamped in a nondissected zone, and retrograde blood cardioplegia was administered.

On opening the aorta, we found a circumferential tear of the intima that had caused the described proximal intussusception. Above the level of the right coronary ostium, the tubular flap was partially lacerated, making residual coronary arterial perfusion still possible. The aorta below the sinotubular junction was spared. Surgical treatment consisted of the complete resection of the dissected portion of the ascending aorta and, after generous mobilization of the aortic arch and of the heart (by freeing the inferior vena cava at its diaphragmatic passage), a direct aortoaortic anastomosis with a 4-0 polypropylene suture. Despite the short duration of the aortic repair, the use of controlled reperfusion, and the absence of bleeding complications (although the patient had received systemic fibrinolysis), weaning from cardiopulmonary bypass was complex as a result of poor left ventricular performance. Cardiac compression by sternal closure was not tolerated; delayed sternotomy closure was therefore necessary. Inotropic requirements decreased from the immediate postoperative period to the 

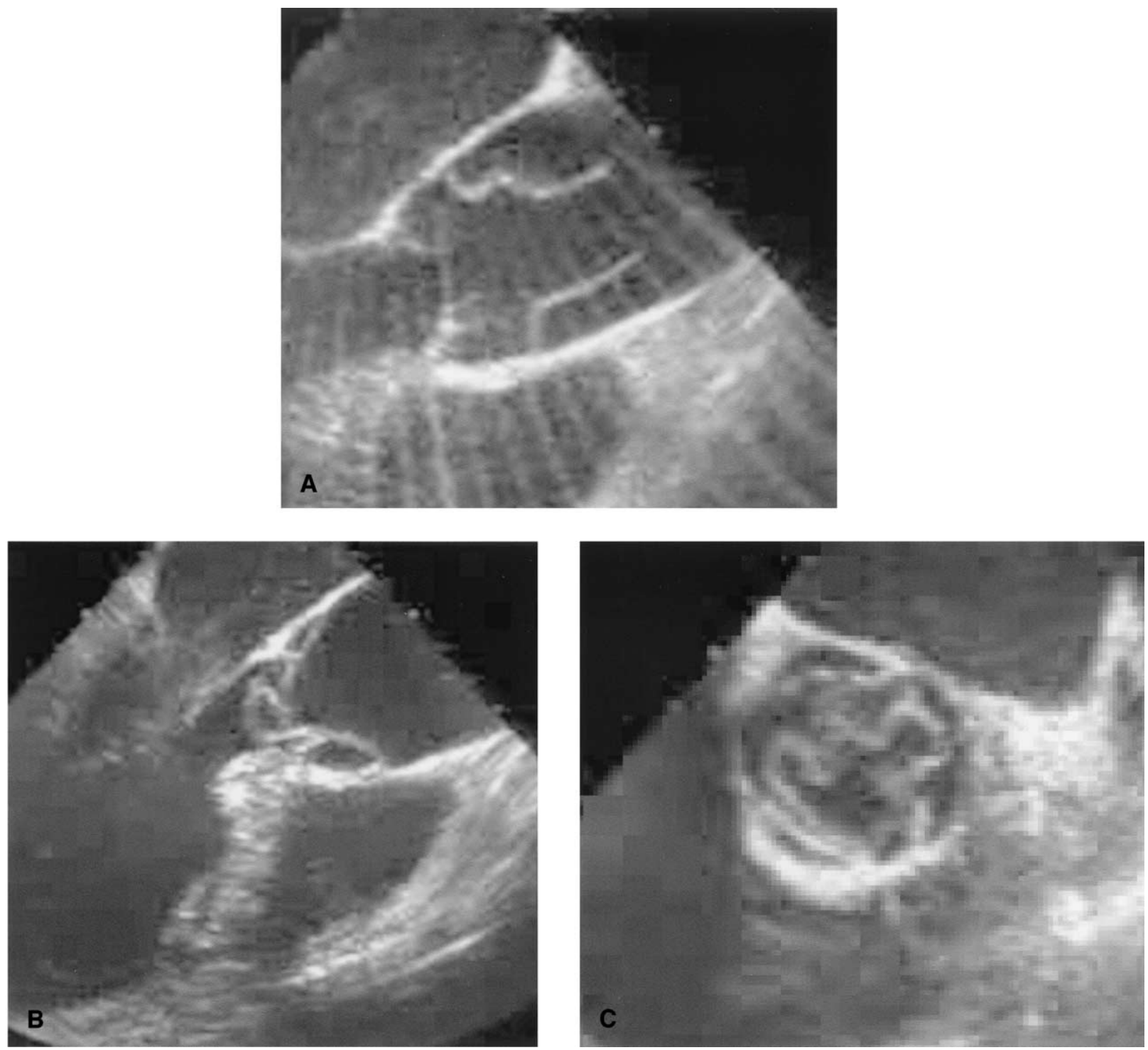

Figure 1. Transesophageal echocardiograms during systole (A) and diastole (B and C) demonstrate proximal invagination through aortic valve, inside left ventricle, of tubular flap, determined flow obstruction of coronary arteries and subsequent myocardial malperfusion.

5th postoperative day just before chest closure (on 6th postoperative day), indicating patient stabilization. Postoperative creatine kinase MB level (peak creatine kinase MB 45 IU/L) returned to normal, and significant recoveries form segmental wall motion abnormalities were observed: left ventricular end-systolic volume decreased from 170 to $120 \mathrm{~mL} / \mathrm{m}^{2}$, left ventricular end-diastolic volume decreased from 99 to $57 \mathrm{~mL} / \mathrm{m}^{2}$, and left ventricular ejection fraction increased from 0.25 to 0.4 .

The subsequent postoperative course was uneventful. The patient recovered well from the operation and was discharged on postoperative day 17. Histologic examination of the aortic wall specimen showed cystic medial necrosis.

\section{Discussion}

In 1962, Hufnagel and Conrad ${ }^{4}$ first described the circumferential disruption of the inner aortic wall layers of the ascending aorta and its subsequent distal invagination. They named this particular presentation of acute aortic dissection intimointimal intussusception. To date, all cases described in the literature ${ }^{5}$ have shown a distal invagination. In our patient the presence of a proximal invagination determined this atypical presentation with coronary malperfusion. In the presence of an established diagnosis of acute aortic dissection, the presence of coronary malperfusion and myocardial ischemia can be easily ruled out by simple diagnostic and 
laboratory investigations. Conversely, aortic dissection is not often considered a cause of myocardial malperfusion; thus misdiagnosis, which represents one of the main preoperative risks for these patients, may frequently occur. ${ }^{1}$ This was the case for our patient, to whom systemic thrombolytic treatment was given before a diagnosis of acute aortic dissection was made.

The subsequent surgical treatment was strongly influenced by this inadvertent treatment, which induced us to avoid any prosthetic material to replace the ascending aorta and imposed a closed technique of anastomosis to avoid hypothermic circulatory arrest. It should be said, however, that the limited longitudinal extension of the dissection was well suited to the repair technique.

This procedure does not fulfill the radical criteria advocated by some authors in the settings of acute type A dissection. ${ }^{6}$ However, it should be underlined that the resection included the entire dissected portion of the aorta and that the parts left in place exhibited a completely normal aspect.

\section{References}

1. Neri E, Toscano T, Papalia U, Frati G, Massetti M, Capannini G, et al. Proximal aortic dissection with coronary malperfusion: presentation, management, and outcome. J Thorac Cardiovasc Surg. 2001;121:55260.

2. Rizzo RJ, Aranki SF, Aklog L, Couper GS, Adams DH, Collins JJ Jr, et al. Rapid noninvasive diagnosis and surgical repair of acute ascending aortic dissection: improved survival with less angiography. J Thorac Cardiovasc Surg. 1994;108:567-75.

3. Borst HG. Organ ischemie. In: Borst HG, Heinemann MK, Stone CD, editors. Surgical treatment of aortic dissection. 1st ed. New York: Churchill Livingstone; 1996. p. 249-64.

4. Hufnagel CA, Conrad PW. Intimo-intimal intussusception in dissecting aneurysm. Am J Surg. 1962;103:723-7.

5. Neri E, Capannini G, Carone E, Tucci E, Diciolla F, Sassi C. The missing flap: considerations about a case of aortic intussusception. J Thorac Cardiovasc Surg. 1999;117:829-30.

6. Westaby S, Saito S, Katsumata T. Acute type A dissection: conservative methods provide consistently low mortality. Ann Thorac Surg. 2002; 73:707-13.

\title{
The frozen elephant trunk technique: A new treatment for thoracic aortic aneurysms
}

\author{
Matthias Karck, MD, ${ }^{\text {a }}$ Ajay Chavan, MD, ${ }^{\text {b }}$ Christian Hagl, MD, ${ }^{a}$ Holger Friedrich, MD, ${ }^{a}$ Michael Galanski, MD, ${ }^{\mathrm{b}}$ and \\ Axel Haverich, MD, ${ }^{a}$ Hannover, Germany
}

ombined diseases of the aortic arch and the descending aorta remain among the great challenges facing cardiothoracic surgeons. In 1983, Borst and associates ${ }^{1}$ introduced the two-stage elephant trunk principle into
cardiovascular surgery, which has greatly facilitated surgical treatment of this special pathologic entity. This approach is based on the prosthetic replacement of the ascending aorta and aortic arch with an elephant trunk extension of the arch graft inserted into the descending aorta during the first-stage operation performed through a median sternotomy. However, the graft segment forming the elephant trunk is floating freely in the descending aortic lumen, thus impeding thrombus formation between the graft and the aneurysmal vessel wall. Because thrombotic occlusion of this space is a prerequisite for reduction of wall stress of the aneurysmal vessel-thereby preventing subsequent growth of the aortic

\footnotetext{
From the Divisions of Thoracic and Cardiovascular Surgery a and Radiology, ${ }^{\mathrm{b}}$ Hannover Medical School, Hannover, Germany.

Received for publication June 26, 2002; accepted for publication July 7, 2002.

Address for reprints: Matthias Karck, MD, Division of Thoracic and Cardiovascular Surgery, Hannover Medical School, Carl-Neuberg-Strasse 1, Hannover 30625, Germany (E-mail: Karck@thg.mh-hannover.de).

J Thorac Cardiovasc Surg 2003;125:1550-3

Copyright $\odot 2003$ by The American Association for Thoracic Surgery $0022-5223 / 2003 \$ 30.00+0$

doi:10.1016/S0022-5223(03)00045-X
}

diameter ${ }^{2}$ - the elephant trunk prosthesis either has to be connected directly to the distal descending portion of the aorta or needs to be extended to the desired level requiring the secondstage operation through a left thoracotomy.

It is evident that comorbidity is a relevant issue in patients with aneurysmal diseases of the aorta, so new therapies to reduce the surgical trauma and risk are desirable. We describe a new approach that allows complete surgical treatment of such combined lesions during a single operation (through a median sternotomy) by using a prototype stent graft that can be placed into the descending aorta in an antegrade fashion through the opened aortic arch.

\section{Methods}

Between September and November 2001, a total of 4 patients were operated on after approval was obtained from the institutional review board of Hannover Medical School. Informed consent was obtained in each case.

In each case spiral computed tomographic angiography of the thoracic aorta was performed before the operation to demonstrate the extent of aortic pathologic process and to determine the correct stent graft size. A custom-made, prototype Chavan-Haverich $(\mathrm{CH})$ stent graft was used for the procedure. The $\mathrm{CH}$ stent graft (Curative Medical Devices Gmbh, Dresden, Germany) consisted of a Dacron polyester fabric vascular prosthesis with stainless steel stents affixed on the inner aspect at its distal end with the help of polypropylene sutures. All stents were fully covered with the Dacron prosthesis. The proximal portion of the $\mathrm{CH}$ stent graft was composed merely of a Dacron tube. The length of the stent graft 